\title{
Politique
}

\section{Les États-Unis : vers un nouvel isolationnisme?}

\section{Philippe Le Prestre}

Numéro 16, automne 1989

Les États-Unis

URI : https://id.erudit.org/iderudit/040633ar

DOI : https://doi.org/10.7202/040633ar

Aller au sommaire du numéro

Éditeur(s)

Société québécoise de science politique

ISSN

0711-608X (imprimé)

1918-6584 (numérique)

Découvrir la revue

Citer cet article

Le Prestre, P. (1989). Les États-Unis : vers un nouvel isolationnisme? Politique, (16), 5-33. https://doi.org/10.7202/040633ar d'utilisation que vous pouvez consulter en ligne.

https://apropos.erudit.org/fr/usagers/politique-dutilisation/ 


\title{
Les États-Unis: vers un nouvel isolationnisme?
}

\author{
Philippe Le Prestre \\ Université du Québec à Montréal
}

De défis en accommodements, l'état d'âme américain s'est cristallisé autour de la notion de déclin. Professeurs et hommes politiques propagent le même message: les obligations militaires ne peuvent continuer à dépasser les ressources économiques sans diminuer la puissance. Selon Robert Gilpin (1981) et Paul Kennedy (1987), une adaptation réussie aux nouvelles conditions internes et externes passe par des réformes économiques intérieures et par la réduction des engagements extérieurs. Selon Mancur Olson (1982), certaines rigidités sociales doivent être surmontées. Robert Keohane (1984), lui, préfère se concentrer sur les conditions favorisant la création ou le maintien de régimes internationaux en l'absence d'un pouvoir dominant pour en faire respecter les règles.

Cet argument ne date pas d'aujourd'hui. Mais l'impact politique de l'essor allemand et japonais, la construction de l'Europe de 1993, et la nouvelle politique soviétique, remettent la problématique de la guerre froide en question et confêrent à cette 
perception une importance nouvelle. Une tendance à exagérer ce que la puissance américaine pouvait accomplir fait progressivement place à une tendance réciproque à sous-estimer l'influence politique qui en découle.

La notion de déclin mérite pourtant un examen attentif (Huntington 1989, Bator 1989). La nature des mécanismes qui, en l'absence de rival multidimensionnel, transforment une relative perte de puissance économique en faiblesse politique croissante, reste obscure. La signification même de ce concept reste vague. Contrairement à des analogies historiques trompeuses, les ÉtatsUnis sont loin de devenir une puissance «ordinaire» (Rosecrance 1976). Le système n'évolue pas d'une hégémonie vers une autre, mais vers une relative diffusion de la puissance. Le terme luimême peut faire référence à une situation tant économique et politique que morale. L'importance de cet argument est avant tout psychologique et réside dans son impact sur la coopération multilatérale et l'exercice du leadership américain.

À quels défis et incertitudes les États-Unis font-ils face? Quels facteurs internes conditionneront leur capacité de les surmonter? Les changements profonds de politique étrangère sont rares et malaisés. Puisque les conditions d'une adaptation réussie à un environnement en mutation se trouvent dans le pays même, une discussions de l'héritage reaganien précédera l'examen de deux importantes variables internes, l'opinion publique et les rapports institutionnels, afin d'éclairer quelques remarques sur la problématique américaine.

\section{L'héritage}

Les politiques étrangères de Kennedy, Nixon et Carter cherchaient toutes à minimiser les effets d'un déclin relatif et à redéfinir le rôle des États-Unis dans le système international. À l'instar de ces dernières, l'Administration Reagan s'efforça de préserver ou de restaurer le statu quo. 


\section{L'environnement externe}

Plus ou moins par dessein, Ronald Reagan a légué à son successeur de réels succès en politique étrangère. De nouveaux problèmes ont, bien sûr, émergé, mais de nombreuses difficultés ont aussi disparu. Que la cause en soit le Président, le Congrès, d'autres pays, ou des forces obscures, ces développements ont à la fois élargi et compliqué la marge de manoeuvre de George Bush.

Le Tiers-Monde, par exemple, a bien changé depuis la fin des années 70. D'une part, la dette et les conditions de vie ont empiré, d'autre part de nouvelles occasions existent de développer des relations politiques constructives. Hormis quelques changements de nature, le volume de l'aide américaine est resté sensiblement le même en dépit du rejet initial de la région. Haîti et les Philippines ont vu le départ de deux dictateurs, sans impact antiaméricain notable. La Corée du Sud s'ouvre lentement et reste stable. Des conflits régionaux ont été résolus ou promettent de l'être (Angola, Namibie, Sahara occidental). L'Union soviétique a retiré ses troupes d'Afghanistan. D'autres pays ont émis quelques tentatives de rapprochement avec l'Occident (Madagascar, Vietnam, Mozambique); même la Lybie s'est modérée. Une vague démocratique a inondé l'Amérique latine, voire le monde entier.

Ces développements furent en partie liés aux déboires soviétiques dans ces régions et à leur volonté de désamorcer ce lieu de compétition. Toutefois, le Nicaragua, le Salvador, et l'Iran posent toujours problème. La stabilité en Amérique centrale passe peut-être par Moscou mais surtout par les États de la région et la modération américaine. L'affaire Iran-Contra, quant à elle, a servi à éduquer le public américain sur la nécessité de restaurer des relations politiques avec ce pays après la mort de l'Ayatollah Khomeyni.

Au Proche-Orient, les États-Unis commencèrent à s'entretenir avec l'OLP à la fin 88 , évitant ainsi à George Bush d'entamer son mandat par une controverse. Le Golfe n'est plus considéré comme le foyer imminent d'un conflit entre les deux Grands. La crise pétrolière semble provisoirement maîtrisée dans deux de ses aspects les plus préoccupants: d'une part l'importance 
des détroits dans le transport du pétrole a diminué, d'autre part les prix restent stables.

Bien sûr, les problèmes économiques et écologiques sont porteurs de catastrophes. La montée des puissances régionales, due en partie à la prolifération nucléaire et au retrait des superpuissances, entraînera un activisme et une insécurité locale aggravés par des querelles séculaires ou idéologiques. En tout état de cause, le Tiers-Monde s'achemine vers une période d'instabilité marquée.

Loin d'être en expansion, l'Union soviétique se retire de sa sphère d'influence. Au lieu de se faire menaçante, elle se veut rassurante. Ronald Reagan, pourtant enclin à la méfiance et convaincu que le conflit entre les deux superpuissances était inévitable, déclara que les réformes de Gorbatchev l'impressionnaient et que les États-Unis devaient miser sur sa sincérité. Un traité révolutionnaire sur les forces nucléaires intermédiaires (FNI) élimina deux fois plus de missiles soviétiques qu'américains, abolit tous les SS4, SS5, et SS20, et adopta des principes de vérification étendus. Même le traité START était presque terminé au moment de l'inauguration de George Bush. Enfin, Ronald Reagan, qui n'avait pas rencontré de dirigeants soviétiques pendant son premier mandat, s'entretint avec le secrétaire général à cinq reprises (plus qu'aucun de ses prédécesseurs à la Maison-Blanche), entérinant ainsi le principe de sommets réguliers entre les deux pays.

Les gains psychologiques furent considérables. Ni les États-Unis ni l'Union soviétique ne pensent que leur position se détériorera tant que l'IDS américaine et les mouvements nationalistes en URSS restent limités. Cela ne peut que renforcer la stabilité de la dissuasion et les négociations sur le contrôle des armements, et permettre à chacun de faire face aux problèmes intérieurs qui le préoccupent. Pourtant, l'avenir des réformes et le caractère futur du régime soviétique suscitent de grandes incertitudes. LURSS deviendra-t-elle plus menaçante et son régime plus séduisant quand son retard économique sera surmonté? Y aura-t-il une réaction non pas Brezhnevienne mais nationaliste et militariste? Sera-t-elle tentée de déstabiliser le Tiers-Monde pour forcer 
une neutralité en Europe ou des accommodements sur ce front central?

Ces questions prennent plus d'importance dans le contexte d'une Alliance en mutation. Militairement, l'OTAN est plus puissante que jamais en vertu de la réintégration indirecte de la France en son sein. Mais politiquement, les divergences d'intérêts entre Américains et Européens se sont accentué. Aux éternelles suspicions à l'égard des intentions américaines s'ajoute une certaine défiance parmi les Européens eux-mêmes. Les dirigeants américains ont cherché à montrer qu'ils avaient la volonté de courir des risques politiques afin de renforcer ou de maintenir la confiance alliée: soutien du Royaume-Uni dans la campagne des Malouines, visite du cimetière allemand de Bitburg, coopération nucléaire avec la France, dénonciation du principe de non-recours en premier à l'arme nucléaire, exclusion des arsenaux nucléaires britanniques et français des négociations sur le contrôle des armements, et déploiement des euromissiles. Mais Ronald Reagan a aussi clairement souligné les divergences d'intérêts fondamentales entre l'Amérique du nord et l'Europe occidentale. Le sommet de Reykjavik, le traité de Washington, et l'IDS ont contribué à la délégitimation des armes nucléaires que la France, le Royaume Uni, et la R.F.A. considèrent indispensables au maintien de la paix. De nouvelles pressions en vue de les réduire davantage se feront sentir au moment précis où des réductions dans l'arsenal conventionnel rendront ces armes plus nécessaires. Enfin, le Japon et la R.F.A. possèdent maintenant des intérêts mondiaux et une puissance économique globale. Ils éprouveront le besoin d'acquérir les moyens militaires et diplomatiques de les protéger alors que les conflits économiques et politiques avec les États-Unis augmenteront, et que toute garantie nucléaire restera inacceptable, trop chère, ou peu crédible.

L'effort de réarmement stratégique a rendu le contrôle des armements acceptable aux conservateurs et a accru les moyens et la volonté d'intervention américaine. Mais les opérations militaires entreprises ont plus conforté l'opinion publique que l'influence des États-Unis. Quoique désireux de restaurer la puissance américaine, Ronald Reagan accepta finalement les 
contraintes internes sur l'emploi de la force qu'il utilisa avec circonspection, selon les voeux du Département de la Défense. Cette prudence fut visible au Liban, au Nicaragua et au Panama, et dans le soutien (pas toujours automatique) aux mouvements de guérilla, sans engagement militaire indéfini. Cette politique a contribué à réconcilier le public américain avec l'usage ponctuel et rapide de la force. Mais elle a également renforcé sa répugnance à soutenir son utilisation prolongée et sans buts clairement définis, attitude qu'illustrent bien les conditions que les chefs d'état-major et Caspar Weinberger cherchèrent à placer sur son emploi après la tragédie libanaise.

L'environnement externe est devenu extrêmement turbulent. La puissance se décentralise fonctionnellement et géographiquement. La croissance économique de l'Europe, du Japon et des nouveaux pays industrialisés accroît les occasions de conflits. Alors que les deux Grands réduisent leurs arsenaux nucléaires, le Royaume-Uni et la France augmentent fortement le leur, et d'autres pays acquièrent des capacités non négligeables. La fin de Yalta a ravivé des conflits ethniques, politiques ou territoriaux séculaires dont les effets peuvent déborder les frontières. Les puissances régionales s'affirment. L'arme nucléaire est en perte de légitimité et les crédits militaires en danger chez la plupart des membres de l'OTAN. Enfin, de nouveaux problèmes transnationaux émergent rapidement: de l'environnement aux réfugiés ou à la drogue. En l'absence de puissance hégémonique, leur résolution se fera grâce à des coalitions transnationales et transgouvernementales difficiles à former.

\section{L'environnement interne}

Sur le plan interne, un des legs les plus significatifs est le contrôle des conservateurs. Ronald Reagan a en fait adopté des politiques auxquelles ces derniers sont traditionnellement opposés. Après la morosité de l'époque Carter, il a restauré la crédibilité de la puissance américaine et une certaine assurance, renforcée par 
l'extinction du mythe de l'irréversibilité des avancées communistes (Tucker 1989).

Mais c'est aussi sur le plan interne que l'héritage est le plus inquiétant. Les tentatives de reconstitution d'une assise interne de la politique étrangère ont manifestement échoué (Tucker 1989). Cela est doublement ironique. D'une part, alors que la sécurité des États-Unis est meilleure, leur capacité d'adaptation a diminué. D'autre part, la problématique reaganienne était avant tout nationale et visait à remédier l'héritage de la guerre du Vietnam et de Watergate, notamment la fin du consensus, la répugnance à utiliser la force, la nécessité d'obtenir le soutien du public à toute politique étrangère, et le besoin de rétablir la confiance dans l'État. À cela s'ajoutait le pessimisme des années Carter provenant de la perception d'une dérive morale associée à l'érosion de la puissance américaine.

Ce consensus est plus absent que jamais, qu'il porte sur l'utilisation de l'arme nucléaire, la justification de l'usage de la force, la politique en Amérique centrale, le contrôle des armements, les relations avec les alliés, le rôle des États-Unis dans le monde, ou celui de l'Exécutif et du Congrès. Ronald Reagan n'a pas essayé d'éduquer le public sur les sacrifices nécessaires au maintien de la puissance américaine ou sur les conséquences de l'évolution du système international. Pire, le président lui-même remit le nucléaire et la dissuasion en question. Le soutien à la Contra, le rejet du Traité sur le droit de la mer, et les critiques du système onusien ont encouragé l'émergence de mouvements politiques transnationaux opposés à l'action du gouvernement. L'affaire Iran-Contra, la réinterprétation du traité ABM, les campagnes de désinformation, les corruptions et autres trafics d'influence ont érodé la crédibilité de l'État et instauré des divisions profondes au sein du gouvernement. Le fossé entre la MaisonBlanche et le Congrès s'est élargi.

Enfin les problèmes économiques et budgétaires (déficits, faillites des caisses d'épargne), et le coût unitaire croissant des nouvelles armes, vont imposer de très fortes limites à la politique de défense. Le budget militaire commença à diminuer en 1985-86, plus en raison du déficit budgétaire qu'à cause de Gorbatchev. La 
question n'est plus de savoir si les dépenses militaires vont diminuer, mais de combien, et quels secteurs seront affectés. Réduire les dépenses comporte maintenant moins de risques politiques après les scandales du Pentagone, les révélations sur le manque de préparation des troupes, et l'offensive de paix soviétique.

Les pressions contre tout engagement financier destiné à aider à la formation d'un nouvel ordre multilatéral seront énormes. Les États-Unis agiront plus comme catalyseurs financiers que comme bailleurs de fonds, limiteront leurs engagements à l'égard du Tiers-Monde et de l'Europe orientale, et devront encourager la Communauté européenne, le Japon et l'Allemagne à jouer un plus grand rôle dans ce processus, au prix de concessions politiques.

\section{La fin des consensus}

Un des grands échecs de la politique reaganienne fut d'avoir aggravé les contraintes internes relatives à l'élaboration de la politique étrangère. Celles-ci ne sont pas nouvelles; chaque secrétaire d'État s'en est plaint (Rusk 1984). Mais elles ont atteint de nouveaux sommets et menacent la capacité de l'État à s'adapter à un environnement extérieur en mutation. La première tâche est d'obtenir le soutien du public. Cette exigence chère à Henry Kissinger et Cyrus Vance, comporte deux aspects: l'un est la relégitimation de la dissuasion, l'autre, la construction d'un nouveau consensus.

\section{L'avenir de la dissuasion}

Le renforcement de la dissuasion est à l'ordre du jour (Brzezinski 1988, Nixon 1989, Huntington 1988). La critique traditionnelle touchait principalement la crédibilité de la dissuasion élargie, que l'adoption de la doctrine de riposte gradué dans les années soixante avait pour but de renforcer. Pourtant, les appréhensions gaulliennes furent confirmées publiquement en 1979 par 
Henry Kissinger dans un discours remarqué, puis lorsque l'OTAN décida de déployer de nouveaux missiles à moyenne portée.

À cette doctrine, il existe un triple défi: politique, technologique et théorique. Le défi politique provient de l'absence ou de la fragilité du soutien à la dissuasion parmi les populations américaines et européennes. La rhétorique reaganienne, initialement axée sur la victoire nucléaire et destinée, en partie, à améliorer la crédibilité de la doctrine, a engendré des mouvements de protestation antinucléaires qui ont ravivé les incertitudes et les suspicions profondes envers ce concept. La vulnérabilité des territoires et des populations est de plus en plus mal ressentie, au moment même où la menace diminue. Alors que sa propre administration contribuait à saper le soutien à la dissuasion en Europe, le président accrut le malaise général, en dénonçant l'immoralité de cette doctrine dans le cadre de son plaidoyer en faveur de l'IDS. Les évêques catholiques lui emboîtèrent le pas.

Enfin, le nouveau contexte de la dissuasion pose le problème de sa pertinence. La dissuasion s'applique-t-elle nécessairement à un environnement moins compétitif? Les techniques de dissuasion, qui enseignent comment gérer une relation hostile, ne risquent-elles de créer des conflits là où il n'en existe pas (Jervis 1979)? Inversement, comment la dissuasion peut-elle contribuer à l'amélioration de ces relations?

Le défi technologique, pour sa part, menace la stabilité de la dissuasion. La modernisation des armements, les problèmes de crédibilité et la dynamique de l'arms control ont conduit à l'adoption d'une stratégie anti-forces. Les deux Grands acquirent, par là même, des capacités de première frappe (MIRVs̀), favorisant une stratégie de préemption en cas de crise. Il existe trop d'armes anti-forces ou indétectables (comme les SLCMs sur l'utilité desquels les militaires américains sont partagés). La capacité de contrôler l'escalade en dominant chaque échelon peut encourager la prise de risques, et la création de «pare-feux» supprimer ce même danger d'escalade, fondement de la destruction mutuelle assurée (Jervis 1984, Kattenburg 1985). L'IDS menace la stabilité en incitant les Soviétiques à conclure que leur position stratégique pourrait empirer, sentiment proche de l'état d'esprit allemand en 
1914, japonais en 1941, ou israélien en 1967 (Brams et Kilgour 1988). Étant donné la réduction d'ampleur de ce programme, son importance réside plus dans sa dimension psychologique que stratégique.

La stabilité est également remise en question par la modernisation des arsenaux nucléaires français et britanniques, laquelle accroîtra la complexité des calculs des superpuissances et les possibilités d'accidents ou d'escalade (Kolodziej 1989). Dans ces conditions, comment renforcer la stabilité? Quels systèmes déployer et annuler? Faut-il abolir les missiles mobiles comme le propose START? La pire solution serait de laisser le Congrès et des intérêts électoraux et bureaucratiques étroits gouverner ces choix stratégiques.

Le troisième défi est plus théorique. Robert Jervis (1979) a souligné le manque de fondement empirique de la dissuasion. Peutelle être aussi étendue aux guerres locales ou aux conflits de faible intensité (George et Smoke, 1989)? Sous quelles conditions une théorie développée dans le contexte des relations stratégiques entre les superpuissances est-elle applicable dans d'autres contextes? Cette question acquiert une importance nouvelle en raison de l'instabilité probable du système international dans les prochaines années.

Enfin, la psychologie a remis le critère de rationalité en question. Certaines tactiques dissuasives peuvent avoir des répercussions négatives, comme l'ont illustré la crise des missiles de Cuba et la politique israélienne à l'égard des pays arabes (George et Smoke 1974; Stein et al. 1987). Selon Richard Lebow et Janice Stein (1989), les facteurs internes et le jeu politique au sein d'une alliance stavèrent cruciaux. Ces auteurs mettent aussi en doute les postulats selon lesquels les décideurs (i) agissent rationnellement, (ii) n'hésitent guère à prendre des risques pour maximiser leurs gains, (iii) sont libres de contraintes internes, et (iv) sont capables de s'identifier clairement comme défenseur ou challenger.

Dans un contexte de riposte graduée et en situation de crise, un acteur peut-il effectuer des calculs raffinés, complexes et exacts, libres de problèmes bureaucratiques, psychologiques, ou liés à la communication? Comme la guerre, la dissuasion commence dans 
l'esprit des hommes; les perceptions affectent donc la rationalité de la décision. La fausse perception des valeurs et des convictions de l'adversaire peut affaiblir, non seulement la dissuasion directe (Jervis 1982), mais aussi la dissuasion élargie, quand chacun est convaincu que la menace d'une intervention peut agir en sa faveur. Une analyse historique rigoureuse a conduit Bruce Russett et Paul Huth (1988) à souligner l'importance du contrôle de l'Exécutif dans le succès de la dissuasion élargie, contrôle affaibli par les interventions du Congrès, les pressions du public, et les conflits bureaucratiques.

À l'approche proposée par le rapport de janvier 1988 sur la Discriminate Deterrence fondée sur le contrôle de l'escalade verticale et horizontale (Armes 1989), il faut substituer une stratégie destinée à contrer, non les capacités, mais la menace, par une politique d'accommodements et d'équilibre. Toutefois, les questions auxquelles ce rapport entendait répondre subsistent: comment réconcilier les intérêts divergents des États-Unis et de l'Europe et relégitimer la dissuasion? Et comment équilibrer ressources et engagements?

Une direction possible consisterait à renforcer le pilier européen et à reprendre le principe de la destruction mutuelle assurée (Jervis 1979). Cette position deviendra plus acceptable après les réductions significatives des arsenaux stratégiques envisagées par START I et de futurs START II, et après la minimisation du rôle des armes nucléaires dans les relations stratégiques. Une telle doctrine est plus stable, moins dépendante du critère de rationalité ou de percées technologiques, et sa crédibilité dépend moins des performances technologiques que de la volonté politique.

L'influence réelle de la théorie sur la politique mise à part, la doctrine de la dissuasion a servi à légitimer les choix de sécurité et à vendre une politique de défense. Sa remise en question contribue à fracturer le consensus des élites et rend le soutien à toute politique plus difficile à construire. 


\section{Vers un nouveau consensus?}

La restauration d'un consensus en politique étrangère constituera une des tâches principales de la nouvelle administration. Walter Lippmann pensait que l'ultime test de toute politique étrangère était son impact sur l'unité du peuple américain (Steel 1981). John Kennedy, comme Wilson et Roosevelt avant lui, chercha à définir une vision globale pour le peuple américain. Jimmy Carter pensait que l'influence diplomatique était à l'image de la force morale intérieure (Carter, 1976). Le souci de l'unité intérieure a, selon Dallek (1983), façonné la politique étrangère américaine tout au long de son histoire.

La conviction qu'une politique étrangère doit non seulement contribuer à la stabilité politique intérieure, mais ne peut réussir que si le peuple américain la soutient, limite la flexibilité des décideurs. Ayant éprouvé la contestation de la guerre du Vietnam, l'incompréhension de son grand dessein stratégique, et l'affaiblissement de l'autorité présidentielle après Watergate, Kissinger a beaucoup insisté sur cette question dans ses mémoires. Aucune politique ne peut réussir sans une certaine congruence entre elle et le public. Cohérence et continuité exigent le soutien d'une majorité stable.

Le problème des présidents Nixon et Carter fut de légitimer des politiques sophistiquées alors que le public américain rejetait leur prémisse, à savoir qu'il existait des limites à la puissance américaine (Williams 1987). Jimmy Carter et Ronald Reagan s'employèrent tous deux à régénérer la foi des Américains dans la politique étrangère de leur gouvernement. George Bush affronte donc un problème commun à chaque administration depuis Nixon: comment, dans un contexte politique en profond changement, reconstruire un consensus détruit par le Vietnam puis par les premières initiatives de son prédécesseur?

Ce fameux consensus de l'après-guerre fut plutôt une majorité stable forgée par la peur et la recherche de la sécurité absolue. Ces deux éléments ont maintenant disparu. Il n'existe plus de stabilité dans l'opinion ni de consensus parmi les élites ou le public. Différentes visions s'opposent, touchant la nature du 
système international, le rôle des États-Unis dans le monde, la doctrine nucléaire, aussi bien que les relations avec l'Union soviétique, l'Amérique centrale, l'Alliance atlantique, le Japon, ou le Tiers-Monde. Ces désaccords se traduisent par des conflits institutionnels, par la paralysie et l'incohérence du Congrès, par la désobéissance civile (affaire Iran-Contra, mouvement sanctuaire), ou par le développement d'initiatives diplomatiques privées.

Comme dans les années trente, les élites semblent profondément divisées (Holsti et Rosenau 1984). On peut distinguer trois attitudes: deux internationalistes (l'une dure, bipolaire et antiaccommodante, l'autre multilatérale et plutôt accommodante) et une semi-isolationniste. Selon cette dernière, un internationalisme excessif conduit à un déclin interne sur les plans politique, économique et social (Idem, Wittkopf 1986). Pour les semi-isolationnistes, les ressources doivent définir les intérêts. Sous la pression des défis économiques, sociaux et politiques qui rendent le leadership diplomatique malaisé, la tentation sera grande, en effet, de maximiser une marge de manoeuvre réduite.

Cette attitude, plus évidente chez le grand public, s'est accrue jusqu'au début des années 80 , quand la plus petite proportion d'Américains depuis 1945 soutenait l'internationalisme (Wittkopf 1986). Cependant, quatre ans plus tard, la proportion favorable à un rôle actif des États-Unis dans le monde passa de $54 \%$ à $70 \%$. En $1986,70 \%$ du public et $85 \%$ des élites étaient favorables à l'augmentation ou au maintien des engagements envers l'OTAN. Mais alors que la volonté d'utiliser les forces armées pour défendre l'Europe et le Japon s'accroît, la résistance à leur utilisation dans d'autres thêâtres continue. Après le réarmement massif des années 80 , le public américain, désireux de maximiser à la fois la paix et la puissance militaire, préfère au schéma de la guerre froide, une politique d'accommodement prudente, la réduction du budget militaire, et un meilleur contrôle des armements (Yankelovich et Smoke 1988; Holsti et Rosenau 1986).

Cela suggère que le retour à la forme ancienne de l'isolationnisme n'est guère probable dans l'immédiat, sans pour autant signifier que le public favorisera une approche multilatérale ou 
mondialiste à long terme. L'ère Reagan a vu la retraite du multilatéralisme en faveur d'un certain unilatéralisme; la remise en question de l'ONU, le rejet du traité sur le droit de la mer et la violation du droit international au Nicaragua en témoignent. Le débat actuel sur le déclin américain est porteur d'une vague néoisolationniste qui conteste le rôle global des États-Unis, leur réseau de bases étrangères, l'aide économique et militaire massive, les systèmes d'alliance formels, ou les concessions unilatérales (Thompson et Zuk, 1986).

Paradoxalement, le désir de forger un consensus limite la capacité du gouvernement de tirer profit des changements propices dans l'environnement international, car il amène souvent à s'en remettre au plus petit dénominateur commun (Jervis 1979). La reconstruction de ce consensus ne peut s'opérer que par l'exploitation habile de développements extérieurs dramatiques, par des débats publics au Congrès, par le leadership de l'Exécutif, et par la coopération entre le Congrès et la Maison-Blanche.

\section{La Maison-Blanche, le Congrès et le syndrome de Madison}

Le rôle que joue le Congrès en politique étrangère comprend deux aspects: (i) le syndrome de Madison, soit l'influence des groupes d'intérêt par l'entremise du pouvoir législatif, et (ii) les relations entre l'Exécutif et le Congrès, soit la question du contrôle de la politique étrangère.

La fin du consensus internationaliste signifie la négation complète de l'aphorisme selon lequel «la politique doit s'arrêter au bord de l'eau». La dépendance politique et financière des élus envers les comités d'action politique a entraîné la domination du processus législatif par des intérêts spécialisés. Fondant la liberté sur le pluralisme d'influence, la formule madisonienne nuit à la capacité d'adaptation car elle empêche la poursuite d'une politique cohérente et soutenue (Cerny 1989). Ce n'est qu'au prix de grandes difficultés que l'État coordonne sa puissance économique, militaire et culturelle en vue d'établir et de faire respecter un ensemble 
consistant de règles et de sanctions. La décentralisation du pouvoir au sein du Congrès, et l'affaiblissement des partis, ont encore accru l'impact des groupes externes.

Comme le montre le domaine de la défense où la coordination budgétaire, stratégique, ou politique est minime, la formule madisonienne de dispersion du pouvoir, au lieu d'encourager l'intégration des intérêts, a servi leur addition. Le président se voit obligé de marchander avec le Congrès, l'administration, les groupes d'intérêts extérieurs et leurs alliés intérieurs, en dehors de toute considération de l'utilité stratégique du système d'armement débattu, ou des objectifs de politique étrangère visés par la politique de défense. Les péripéties des différents systèmes de missiles mobiles illustrent ce phénomène.

Mais la capacité de blocage de ces groupes est souvent plus forte que leur capacité d'initiative. Kabashima et Sato (1986) indiquent que la nature des liens des représentants avec les syndicats (plus que leur appartenance politique) n'influença leur vote sur le projet de loi sur le commerce des produits automobiles que lorsque son adoption devint peu probable. Les élus purent alors obliger leurs électeurs et leurs bienfaiteurs tout en envoyant un message aux Japonais. Le fameux «lobby juif» n'a pas empêché l'adoption de politiques contraires aux souhaits d'Israël ou à ceux de la majorité des électeurs juifs, comme en témoignent les relations avec l'OLP. Bien que l'influence de groupes comme l'AIPAC (Arab-Israel Public Affairs Committee) soit quelquefois indéniable, elle n'explique pas la politique américaine. Même dans le domaine de l'aide économique et militaire, leur pouvoir de blocage n'est pas absolu: une opposition massive n'a pu empêcher des livraisons d'armes aux pays arabes sous Carter et Reagan. Ces groupes peuvent influencer le calendrier de certaines décisions, mais la plupart d'entre elles ne résultent pas uniquement de leurs activités (Spiegel, 1985; Bard, 1988). Celles-ci peuvent néanmoins endommager les relations bilatérales.

Dans le cas de l'industrie militaire, des coalitions de puissants intérêts bureaucratiques, législatifs et industriels sont capables d'imposer, sinon son initiation, du moins la continuation d'un programme (Adams 1981, Smith 1988). Les élus ont leurs 
programmes favoris et des emplois à protéger, les civils et militaires leurs carrières, les services leurs rivalités, les industriels leurs bénéfices. La restauration par le Congrès, en 1989, de programmes annulés par le Département de la Défense fournit un exemple récent de la difficulté à mettre en pratique une politique d'acquisitions cohérente.

Lorsque l'historien Arthur Schlesinger dénonçait «la présidence impériale» en 1973, les critiques principales portaient sur les opérations militaires, les pouvoirs d'exception, l'abus des accords exécutifs directs (Executive Agreements), les activités clandestines de la CIA, ou sur des opérations de politique étrangère secrètes et privées. Après Watergate et les réactions du Congrès à l'échec vietnamien, les présidents commencèrent à se plaindre de leur faiblesse.

Aujourd'hui, tout le monde se plaint. Ronald Reagan déplora les restrictions imposées par le Congrès, l'incohérence de ses décisions, et sa tendance à «micro-gérer» la politique. Celui-ci se plaignit du manque d'informations, des défauts de consultation, et des activités illégales de l'Exécutif. Les deux branches éprouvent de plus grandes difficultés à contrôler le processus d'élaboration et de mise en oeuvre de la politique étrangère. Puisque ce conflit reflète le manque de consensus et la complexité croissante de l'environnement extérieur, il ne pourra que se prolonger.

L'opposition du Sénateur Fulbright à la guerre du Vietnam dans les années soixante signala une défiance grandissante du Congrès envers l'Exécutif, alliée au sentiment qu'une certaine abdication législative fut en partie responsable de cette débâcle. Les tentatives de regagner une certaine égalité commencèrent en 1970, avec l'abrogation de la résolution du Golfe du Tonkin de 1964, suivie de nombreux textes visant à limiter la flexibilité de la Maison-Blanche, à inclure le Congrès dans l'élaboration des décisions, et à imposer certaines préférences.

Au lieu de clarifier les choses, les auditions sur l'affaire Iran-Contra en 1987, et le débat sur la candidature malheureuse du juge Bork à la Cour suprême en 1988, ont ravivé la controverse entre les partisans de l'égalité du Congrès et ceux de la primauté de l'Exécutif. L'examen des candidats est devenu plus serré et 
politique, atteignant des sommets au début du mandat de George Bush. Pervertissant cette procédure, des sénateurs comme Jesse Helms, se servirent du processus de nomination de candidats afin de faire pression sur la politique du gouvemement de leur propre parti. En l'absence de critères et règles clairs, l'examen des nominations se politise et permet de s'ingérer dans les décisions présidentielles.

Bien que les conflits entre les deux branches soient profonds, le jeu politique n'est pas limité à leurs interactions. Dans bien des cas, les lignes de partage se situent ailleurs et varient selon les coalitions formées par des éléments de l'administration, de la Maison-Blanche, et du Congrès. Ces coalitions inter-institutionnelles en faveur d'un certain ordre du jour politique comprennent souvent des éléments extérieurs, voire étrangers dans le cas des négociations sur le contrôle des armements. Le personnel législatif peut s'allier avec certains éléments de l'administration ou même promouvoir, seul, son point de vue. Le personnel de la Commission des Affaires étrangères de la Chambre des Représentants trouva des alliés dans la bureaucratie pour avancer le principe de l'aide conditionnelle au respect des droits de l'homme. Certains rejoignirent ensuite l'administration Carter pour mettre en ceuvre sa politique humanitaire (Newsom 1986).

La décentralisation du processus de prise de décision au sein đu Congrès a facilité cette évolution. À la suite de la réduction des pouvoirs des présidents de commission dans les années 70 , les sous-commissions devinrent de nouveaux centres de pouvoir dotés de leur personnel et budget propres, et d'une compétence bien définie. Leurs présidents se sont transformés en entrepreneurs cherchant à contrôler des domaines étroits de la politique étrangère sans que les présidents de commission puissent coordonner ou arbitrer leurs activités. Même une défaite en commission peut se rattraper en session plénière où les règles gouvernant les débats sont maintenant plus ouvertes. De plus en plus d'amendements provenant de membres extérieurs aux commissions compétentes sont introduits et adoptés en session plénière grâce à la constitution de coalitions ad hoc. Progressivement, l'individu, plus que 
la commission ou la sous-commission, devient l'acteur principal (Hammond 1986).

Le manque de consensus et la décentralisation du pouvoir ont permis à certains de se construire des fiefs électoraux. Le représentant démocrate Stephen Solarz, par exemple, put transformer la sous-commission des Affaires asiatiques et pacifiques qu'il préside, en un puissant instrument de financement et de soutien politique (Alston 1989). Fort de cette position, il n'hésita pas à s'opposer à la Maison-Blanche en 1985-86 et à encourager la démission de Ferdinand Marcos. Bien que l'importance relative des questions de politique étrangère dans les résultats électoraux soit discutable, la visibilité qu'elles procurent est évidente et permet quelquefois de se libérer du critère d'ancienneté pour accéder à des fonctions importantes au sein du Congrès.

De plus, certains présidents de commission ont acquis une expertise de loin supérieure à celle d'un secrétaire. Si l'action politique du président ou du secrétaire à la Défense semble vaciller ou hésiter, Les Aspen ou Sam Nunn, à la tête des commissions des Forces armées de la Chambre et du Sénat, n'hésiteront pas à combler le vide. Mais eux-mêmes ne pourront pas toujours compter sur le soutien inconditionnel d'une institution prompte à se rebeller. Les scandales du Pentagone ont aussi avivé la tendance de ces commissions à «micro-gérer» la politique de défense.

Cette évolution est complétée par la propension croissante des dirigeants du Congrès à enquêter, et même à négocier directement avec des dirigeants étrangers. Les exemples se sont multipliés depuis l'Administration Ford. Sous Reagan, Richard Helms pour l'aile conservatrice, et Jim Wright, président démocrate de la Chambre, menèrent chacun leur propre politique en Amérique centrale. Richard Lugar, alors président républicain de la commission sénatoriale des Affaires étrangères, força la main du président et obtint le départ de Ferdinand Marcos. Des gouvernements étrangers ont, à leur tour, essayé d'influencer la position de certains membres du Congrès sur les ventes d'armes. Ces contacts peuvent avoir un effet positif lorsqu'ils améliorent la compréhension des réalités locales, mais la répétition d'auditions et de dépositions directes (souvent arrangées par des groupes 
américains alliés à des groupes étrangers) soulève certaines questions relatives à la coordination et à la manipulation du processus politique. À tout le moins, cette transnationalisation de la politique étrangère complique grandement la définition et la mise en oeuvre de toute politique.

Enfin, Ronald Reagan lui-même a détruit le peu de confiance qui subsistait entre les deux branches, non seulement en raison de l'affaire Iran-Contra, mais aussi à travers sa rhétorique populiste anti-Congrès, ses tentatives de mettre le Congrès dans l'embarras sur les questions budgétaires, et la réinterprétation du traité $A B M$ dans le cadre de sa campagne en faveur de l'IDS.

Tout cela affaiblit la capacité du président à innover et à appliquer une politique cohérente alors que ces exigences sont plus indispensables que jamais. Une politique prudente, dissociant idéologie et moralité des intérêts politiques à long terme, sera plus difficile à mettre en oeuvre comme en témoigna l'opposition du Congrès à la politique de l'Administration Bush envers la Chine et l'Europe orientale en 1989. Sans leadership présidentiel, le Congrès et la bureaucratie agissent en toute liberté, chacun appliquant sa propre politique étrangère. Seul un Conseil national de sécurité fort peut remédier à cet état de fait.

L'influence d'une grande puissance (et la stabilité du système intemational) ne résident pas dans la capacité de créer une zone d'incertitude autour de ses propres actions, mais proviennent de sa fiabilité et de sa vision à long terme. D'autres pays essaieront de s'accommoder des contradictions, des incertitudes et des faits accomplis tant qu'ils auront besoin des ressources et du soutien américain. Mais une moindre dépendance ou l'érosion de leur confiance les incitera à poursuivre leurs intérêts sans les États-Unis - voire à leur encontre -, isolant ainsi ces derniers davantage.

\section{Choix et tendances}

Selon les tenants d'une approche mondialiste (Hoffman, 1978) ou multilatérale (Steinbrunner 1989), les États-Unis 
devraient coopérer avec les nouveaux centres de puissance afin de construire un nouvel ordre mondial fondé sur des régimes internationaux développés en coordination avec leurs alliés. Certains embrassent une multipolarité qui, en éliminant le carcan bipolaire, permettrait aux États-Unis d'assumer une responsabilité spéciale dans la définition d'un nouvel ordre du jour international autour de l'environnement, de la stabilité du Tiers-Monde, du développement économique, et des valeurs démocratiques (Newsom 1988). Ils regagneraient ainsi la force morale et intellectuelle perdue au cours de la guerre froide.

Dans l'immédiat, le problème reste celui de Kissinger qui, rappelons-le, tenta d'adapter le pays à un déclin, symbolisé alors par la parité nucléaire: comment augmenter la légitimité du système auprès des nouvelles puissances tout en renforçant sa stabilité? D'autres facteurs économiques se sont, depuis, ajoutés à cette évolution structurelle et militaire. La capacité et la volonté des États-Unis d'agir seuls ou de soutenir financièrement de nouveaux régimes diminuent. Peuvent-ils faciliter la transition vers un monde multipolaire? Dépourvus des moyens dont ils disposaient après la guerre, quels aspects leur participation pourrait-elle prendre?

Un jeu multipolaire limité embrasserait d'abord une nouvelle détente axée sur le contrôle des armements et la coopération sur les problèmes régionaux, afin de libérer des ressources pour résoudre les problèmes intérieurs. Conscients de leur impuissance à accélérer seuls l'évolution démocratique, connaissant les racines locales de l'instabilité internationale et la complexité des problèmes de développement, les États-Unis adopteront un activisme limité. Des ressources réduites et des priorités variables viendront freiner, aussi bien la définition et la mise en oeuvre d'un ordre du jour multilatéral qu'ils pourraient soutenir financièrement, que leur participation à de tels arrangements. Invitant d'autres pays à se tourner vers le reste du monde, ils concentreront davantage leur aide dans des régions proches de leur territoire ou touchant l'intérêt électoral d'hommes politiques influents. Enfin, les États-Unis essaieront de convaincre leurs alliés d'assumer une plus grande part du fardeau économique de l'Alliance, au prix de concessions poli- 
tiques et militaires. Dans cet esprit, ils pourraient chercher à constituer, non pas des puissances régionales dominantes (comme l'entendait la doctrine Nixon), mais des équilibres régionaux en Europe et en Asie.

Cela entraînera une redéfinition de l'intérêt national vis-àvis de l'Europe, de l'Union soviétique et du Japon, son extension sélective à de nouveaux domaines comme l'environnement, la dette, ou la drogue (Maynes 1989, Mathews 1989), et sa formulation en termes d'économies budgétaires. Il faudra rassurer l'Union soviétique, incorporer le Japon et l'Europe dans la direction du système mondial (le Japon faisant contrepoids à l'Europe et fournissant aide économique ou même protection militaire à des pays tiers), contrôler l'impact des conflits commerciaux et des problèmes transnationaux, et prévenir le débordement des conflits locaux.

Des pressions économiques, structurelles, et internes forceront les États-Unis à diminuer leur participation à l'OTAN, dont l'avenir passe par la constitution d'un pilier nucléaire européen et la redéfinition de son mandat. Mais la constitution d'une défense européenne viable exigera une volonté américaine de protéger les arsenaux nucléaires européens (impliquant, par exemple, des transferts de technologie pour assurer leur invulnérabilité), au risque de compromettre l'avenir des négociations post-START I. Il faudra encourager la coopération politique et militaire entre la France et la Grande Bretagne, et définir un nouveau rôle pour l'Allemagne dans un contexte de suspicions mutuelles, de dénucléarisation psychologique, de contraintes budgétaires importantes dans la plupart de ces pays, et de pressions soviétiques continuelles.

Dans un monde multipolaire, les incertitudes seront plus nombreuses et les menaces sur la sécurité des États-Unis plus imprévisibles. Un effort d'adaptation exige des moyens d'intervention puissants, donc la consolidation de la puissance navale américaine. La structure des forces américaines devrait alors changer. Au lieu de se préparer à conduire une guerre et demie, les États-Unis devraient sans doute en préparer trois demies, conduites en coopération avec des alliés régionaux. Mais le maintien des 
capacités navales se heurte à des obstacles budgétaires, interarmes, et politiques, alors que l'Union soviétique fait campagne en faveur de leur réduction.

La construction d'un tel système incluant l'Union soviétique, obligera à un dialogue permanent afin de s'assurer qu'aucune des deux parties ne profite de la situation ou n'amplifie les problèmes qu'affronte l'autre. Ces deux pays ont des intérêts communs: éviter une guerre nucléaire, accomplir des réformes internes, réduire leurs obligations à l'étranger sans créer d'instabilité ou favoriser l'autre Grand, et contrôler la prolifération nucléaire. Mais les États-Unis feront face à de nombreux dilemmes entre l'amélioration de leurs relations avec les Soviétiques et un jeu multipolaire. La stabilisation de la phase de transition exigera aussi un ralentissement des politiques réformistes soviétiques: il n'est pas dans l'intérêt immédiat des États-Unis que l'URSS se retire trop vite de certaines régions, y compris d'Europe centrale.

En dépit du rapprochement américano-soviétique, l'environnement international devient dangereux. Les occasions de fausses perceptions entre les Grands vont se multiplier alors que chacun affronte des problèmes internes et externes plus complexes. De nouvelles puissances vont s'affirmer politiquement et militairement. D'anciens conflits ethniques et territoriaux vont resurgir et risquent de déborder. Dans ce contexte, leadership politique intérieur, cohésion, vision, et continuité sont indispensables si les États-Unis veulent pouvoir influencer cette transition multipolaire dans un sens conforme à leurs intérêts et à ceux de leurs alliés.

Le public américain pourra-t-il distinguer entre puissance et contrôle, direction et domination, stabilité et statu quo, efficacité et présidence impériale (Hoffmann 1978)? Pour être viable, une telle politique devra être énoncée dans le contexte d'une nouvelle mission des États-Unis dans le monde. Si quelques questions globales comme l'environnement peuvent y contribuer, la sélectivité des choix sera difficile à justifier. De plus, une politique comprenant la création de pôles régionaux et un jeu d'équilibre sophistiqué associé à la non-intervention en Europe centrale, en Union soviétique ou en Asie (Chine), sera difficile à articuler. La tentation isolationniste sera donc forte, car elle permettrait de 
minimiser l'impact de l'interdépendance sur la société américaine (Ravenal 1985, Kennan 1977), et de contrôler les contraintes intérieures sur la politique étrangère (Ravenal 1975), pour reconstruire la cité sur la colline, symbole et objet renouvelé des espérances humaines.

\section{Conclusion}

Depuis Tocqueville, on déplore les obstacles qu'un système démocratique présente au développement et à la mise en oeuvre d'une politique étrangère efficace. Que ce soit George Kennan ou Ed Rowny, les diplomates américains critiquent sans cesse la perspective à court terme de leurs gouvernements, par opposition à la politique supposée à long terme de l'Union soviétique. D'autres soulignent l'impossibilité de maintenir longtemps un certain cap, le manque de discrétion d'une société ouverte, et le blocage de la société dû au syndrome de Madison. À ces problèmes s'ajoute un système où l'on trouve plus d'hommes politiques professionnels au Congrès qu'à la Maison-Blanche ou à la tête des départements, où il y a pénurie de hauts fonctionnaires compétents, et où les pouvoirs sont partagés plutôt que séparés. La taille et la bureaucratisation de l'administration de la politique étrangère engendrent l'incohérence, et encouragent les compromis et l'inertie aux dépens de l'innovation.

Dans un tel système, le leadership présidentiel est essentiel. Un président n'est puissant que lorsqu'il en a la réputation. La capacité d'adaptation interne et externe dépendra de l'image que le président peut projeter. Cette faculté, à son tour, influence la perception de la position des États-Unis dans le monde. En 1986 par exemple, le public et les élites considéraient que les États-Unis jouaient un plus grand rôle mondial qu'en 1982. Ce sentiment n'était pas simplement dû à un changement dans l'équilibre des forces militaires, mais aussi au style présidentiel. Afin de légitimer sa politique, un président doit projeter une compétence et une vision correspondant aux aspirations américaines traditionnelles. C'est cette vision qui fait défaut. Les hommes de George 
Bush semblent s'entendre (finis les conflits entre Zbigniew Brzezinski et Cyrus Vance, ou entre Caspar Weinberger et George Shulz), mais manquent de vision stratégique ou politique.

Le leadership sera nécessaire afin de contrôler des attentes millénaristes concernant le déclin du communisme et la fin de la rivalité est-ouest, l'avènement d'un libéralisme universel et bienveillant, ou la tendance à la dénucluéarisation. Le système devenant plus dangereux en raison des incertitudes liées au retrait des superpuissances, de la diffusion de la puissance, des possibilités de fausses perceptions, et de la résurgence de mouvements locaux ou régionaux, il est important de renforcer les conditions de stabilité des crises, et de minimiser la capacite de lancer des actions de préemption (Steinbrunner 1989). Si le système en formation ne repose plus sur la dominance d'un pays qui pourrait en contrôler le fonctionnement, les États-Unis continueront à influencer les autres pays plus que ceux-ci ne les influenceront, et pourront contribuer de manière significative à la construction et à la stabilité d'un tel système.

Mais leur capacité politique, économique et institutionnelle d'accomplir la transition multipolaire est très réduite alors que les exigences internes se font de plus en plus pressantes. Au-delà du rôle des États-Unis dans le monde et de leur aptitude à construire un nouvel ordre mondial, le débat sur le déclin américain porte aussi sur la corruption des idéaux, de l'économie, du processus politique, et de la société engendrée par l'internationalisme. Le même souci de renaissance morale se retrouve chez certains mondialistes comme Newsom et chez des néo-isolationnistes comme Kennan. Pour les premiers, cette renaissance passe par un acte de contrition international et un engagement sélectif, pour les seconds, par un aveu d'impuissance et un retour aux aspirations américaines historiques.

Au lieu d'être une aberration passagère, le crépuscule de l'internationalisme des années 80 (Hughes 1985) annonça-t-il l'abandon définitif de l'approche de l'Administration Carter centrée sur l'adaptation à l'interdépendance dans le bien commun? L'amélioration apparente des rapports internationaux fournira à la réaction isolationniste un argument auquel un gouvernement incapable 
d'élaborer une stratégie faisant appel aux valeurs américaines traditionnelles, et soumis à de fortes pressions internes et transnationales, pourra difficilement résister. Le déterminisme économique d'un Kennedy s'associerait alors au pessimisme d'un Kennan et à la déclaration unilatérale de victoire idéologique d'un Fukuyama (1989), pour justifier la quête solitaire d'une nouvelle innocence. 


\section{Bibliographie}

ADAMS, Gordon. The Politics of Defense Contracting: the Iron Triangle, New York, Council on Economic Priorities, 1981.

ALSTON, Chuck, «Solarz Looks Abroad to Find Election Cash at Home», Congressional Quarterly, 47, 10, march 11, 1989, p. 501-4.

ARMES, Keith, «Discriminate Deterrence: Western European Comment», The Atlantic Community Quarterly, p. 247-69.

BARD, Mitchell, "The Influence of Ethnic Interest Groups on American Middle East Policy", in Charles W. Kegley, jr. and Eugene R. Wittkopf, eds. The Domestic Sources of American Foreign Policy, New York, St Martin's, 1988, p. 57-69.

BATOR, Francis, «Must We Retrench?», Foreign Affairs, 68, 2, p. 93-123.

BRAMS, Steven J. and Kilgour, D. Marc, «Deterrence versus Defense: a Game-Theoretic Model of Star Wars", International Studies Quarterly, 32, 1, 1988, p. 3-28.

BRZEZINSKI, Zbiniew, "America's New Geostrategy", Foreign Affairs, 66, 4, 1988, p. 680-699.

CARTER, James E., «Presidential Debate, Oct. 7», 1976.

CERNY, Philip, «Political Entropy and American Decline», Millenium, 18, 1, 1989, p. 47-63.

DALLEK, Robert, The American Style of Foreign Policy, New York, Knopf, 1983.

FUKUYAMA, Francis, «The End of History?», The National Interest, 16, 1989, p. 3-18.

GEORGE, Alexander L. et Smoke, Richard. «Deterrence and American Foreign Policy», World Politics, XLI, 2, 1989, p. 170-182.

GEORGE, Alexander L., Deterrence in American Foreign Policy, New York, Columbia University Press, 1974.

GILPIN, Robert, War and Change in World Politics, Cambridge, Cambridge University Press, 1981.

HAMMOND, Susan Webb, "Congress in Foreign Policy", in Edmund Muskie, Kenneth Rush et Kenneth Thompson, eds. The President, the Congress and Foreign Policy, Lanham, MA: University Press of America, 1986, p. 67-91.

HOFFMANN, Stanley, Primacy or World Order: American Foreign Policy since the Cold War, New York, McGraw Hill, 1978.

HOLSTI, Ole R. et Rosenau, James N., «The Foreign Policy Beliefs of American Leaders: Some Further Thoughts on Theory and Method», International Studies Quarterly, 30, 4, 1986, p. 473-84. 
HOLSTI, Ole R., American Leadership in World Affairs: Vietnam and the Breakdown of Consensus, London and Boston, Allen \& Unwin, 1984.

HUGHES, T.L., «The Twilight of Internationalism», Foreign Policy, ${ }^{\circ} 61,1985-6$, p. $25-48$.

HUNTINGTON, Samuel P., "Coping with the Lippmann Gap», Foreign Affairs, 66, 3, 1988, p. 453-77.

HUNTINGTON, Samuel P., «The U.S.: Decline or Renewal?», Foreign Affairs, 67, 2, 1989, p. 76-96.

JERVIS, Robert, "The Madness Beyond MAD: Current American Nuclear Strategy", $P S, 16,1,1984$, p. 33-40.

JERVIS, Robert, «Deterrence and Perception», International Security, 7, 1982, p. 3-30.

JERVIS, Robert, "Why Nuclear Superiority Doesn't Matter», Political Science Quarterly, 94, 4, 1979-80, p. 617-33.

JERVIS, Robert, «Deterrence Theory Revisited», World Politics, XXXI, 2, 1979, p. 289-324.

JERVIS, Robert, Perception and Misperception in International Politics, Princeton, Princeton University Press, 1976.

KABASHIMA, Ikuo et SATO, Hideo, «Local Content and Congressional Politics: Interest-group Theory and Foreignpolicy Implications», International Studies Quarterly, 30, 3, 1986, p. 295-314.

KATTENBURG, Paul, «MAD is the Moral Position», in Charles Kegley and Eugene Wittkopf, eds. The Nuclear Reader. Strategy Weapons, War, New York, St Martin's, 1985.

KENNAN, George F., The Cloud of Danger, Boston, Little Brown, 1977.

KENNEDY, Paul, The Rise and Decline of the Great Powers, New York, Random House, 1987.

KEOHANE, Robert, "Alliances, Threats, and the Uses of Neorealism», International Security, 13, 1, 1988, p. 169 176.

KEOHANE, Robert, After Hegemony: Cooperation and Discord in the World Political Economy, Princeton, Princeton University Press, 1984.

KOLODZIEJ, Edward A., «British-French Nuclearization and European Denuclearization: Implications for US Policy», in Philippe Le Prestre, ed. French Security Policy in a Disarming World, Boulder, Lynne Rienner, 1989, p. 105-46.

LEBOW, Richard N. et Stein, Janice G., «Rational Deterrence Theory: I Think, Therefore I Deter», World Politics, XLI, 2, 1989, p. 208-24. 
MATHEWS, Jessica Tuchman, «Redefining Security», Foreign Affairs, 68,2, 1989, p. 162-177.

MAYNES, Charles William, «Coping with the 90's», Foreign Policy, ${ }^{\circ} 74,1989$, p. 42-62.

NEWSOM, David D., "The New Diplomatic Agenda: Are Governments Ready?», International Affairs, 65, 1, 1988, p. $29-42$.

NEWSOM, David D., «The Executive Branch in Foreign Policy», in Edmund Muskie, Kenneth Rush et Kenneth Thompson, eds. The President, the Congress and Foreign Policy, Lanham, MA, University Press of America, 1986, p. 93-119.

NIXON, Richard, "American Foreign Policy: the Bush Agenda» Foreign Affairs, 68, 1, 1989, p. 199-219.

OLSON, Mancur, The Rise and Decline of Nations, New Haven, Yale University Press, 1982.

RAVENAL, Earl C., «Europe without American», Foreign Affairs, $63,5,1985$, p. $1020-35$.

RAVENAL, Earl C., «Who Needs It?» Foreign Policy, n 18, 1975 , p. 79-91.

ROSECRANCE, Richard N., ed., America as an Ordinary Country: US Foreign Policy and the Future, Ithaca, Cornell University Press, 1976.

RUSK, Dean, «Remarks», New York Times, Feb. 11, 1984.

RUSSETT, Bruce, et HUTH, Paul, «Deterrence Failure and Crisis Escalation», International Studies Quarterly, 32, 1, 1988, p. 29-45.

SMITH, Hedrick, The Power Game. How Washington Works, New York, Random House, 1988.

SPIEGEL, Steven, The Other Arab-Israeli Conflict, Chicago, University of Chicago Press, 1985.

STEEL, Ronald, Walter Lippmann and the American Century, New York, Vintage, 1981.

STEIN, Janice Gross, LEBOW, Richard Ned, JERVIS, Robert, Beyond Deterrence, Ithaca, Cornell University Press, 1987.

STEINBRUNNER, John D., ed., Restructuring American Foreign Policy, Washington, Brookings Institution, 1989.

THOMPSON, William R. et ZUK, Gary, «World Power and the Strategic Trap of Territorial Committments» International Studies Quarterly, 30, 3, 1986, p. 249-67.

TUCKER, Robert W., «Reagan's Foreign Policy», Foreign Affairs, $68,1,1989$, p. 1-27.

WITTKOPF, Eugene R. «On the Foreign Policy Beliefs of the American People: a Critique and Some Evidence», International Studies Quarterly, 30, 4, 1986, p. 425-45. 
WILLIAMS, Phil, «The Limits of American Power: from Nixon to Reagan», International Affairs, 63, 4, 1987, p. 575-88.

YANKELOVICH, Daniel et SMOKE Richard, «America's New Thinking», Foreign Affairs, 67, 1, 1988, p. 1-17. 